\title{
FINDRISC Modified for Latin America as a Screening Tool for Persons with Impaired Glucose Metabolism in Ciudad Bolívar, Venezuela
}

\author{
María Carolina Muñoz-González ${ }^{\mathrm{a}}$ Marcos M. Lima-Martínez ${ }^{\mathrm{a}, \mathrm{b}} \quad$ Aura Nava ${ }^{\mathrm{a}}$ \\ Gisuardo Trerotola $^{a}$ Mariela Paoli ${ }^{c}$ Julio O. Cabrera-Rego ${ }^{d}$ Briggytte Gonzalez ${ }^{\mathrm{e}}$ \\ Amilcairy Arciniegas ${ }^{\mathrm{e}}$ Javier Paez ${ }^{\mathrm{e}}$ \\ a Department of Physiological Sciences, Universidad de Oriente, Ciudad Bolívar, Venezuela; ${ }^{b}$ Endocrinology,

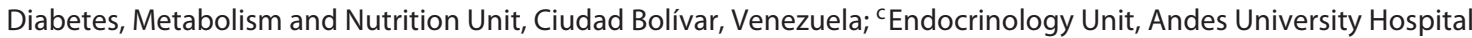 \\ Autonomous Institute, Mérida, Venezuela; 'Intensive Coronary Care Unit, Hospital "Comandante Manuel Fajardo," \\ Havana, Cuba; e Paez Laboratory, Ciudad Bolívar, Venezuela
}

\section{Significance of the Study}

- There are no previous studies that have evaluated the utility of LA-FINDRISC in the southeastern region of Venezuela. A LA-FINDRISC $>14$ points predicts diabetes mellitus or glucose metabolism disorders with a sensitivity of $45.2 \%$ and a specificity of $89.9 \%$. Patients with LA-FINDRISC $>14$ points must undergo an oral glucose tolerance test.

\section{Keywords}

FINDRISC · LA-FINDRISC · Diabetes · Prevention ·

Prediabetes

\begin{abstract}
Objective: The Finnish Diabetes Risk Score (FINDRISC) includes anthropometric, metabolic, and lifestyle factors that predict type 2 diabetes mellitus. The objective of this study was to evaluate the FINDRISC modified for Latin America (LA-FINDRISC) as a screening tool for persons with impaired glucose metabolism in Ciudad Bolívar, Venezuela. Methods: Subjects aged between 18 and 70 years of both sexes without known diabetes were invited to participate. After informed consent, they were screened with the LA-FINDRISC questionnaire and then given an oral glucose tolerance test, using the American Diabetes Association criteria for diagnosis. To obtain the cutoff point of LA-FINDRISC for predicting impaired glucose regulation, a re-
\end{abstract}

ceiver operating characteristic curve was constructed. Results: A total of 200 subjects were evaluated, $64.5 \%$ female, with a mean age of $35.20 \pm 13.84$ years. Of these, 158 (79\%) did not present with carbohydrate metabolism disorder, while 42 (21\%) did. Age $(p=0.0001)$, body mass index $(p=0.011)$, and waist circumference ( $p=0.031$ ) were significantly higher in subjects with impaired glucose regulation when compared to those without it. There were a significantly greater number of sedentary $(p=0.039)$ and hypertensive subjects $(p=0.0001)$, as well as those with a history of glucose $>100 \mathrm{mg} / \mathrm{dL}$ ( $p=$ 0.0001 ), in the impaired glucose metabolism group. A cutoff LA-FINDRISC of 14 points predicted a high risk of impaired glucose regulation with a sensitivity of $45.2 \%$ and a specificity of 89.9\%. Conclusion: A LA-FINDRISC > 14 points had low sensitivity but high specificity for predicting carbohydrate metabolism disorders in this group of patients from Ciudad Bolívar.

(c) 2019 The Author(s)

Published by S. Karger AG, Basel

\begin{tabular}{|c|c|c|}
\hline KARGER & $\begin{array}{l}\text { (c) } 2019 \text { The Author(s) } \\
\text { Published by S. Karger AG, Basel }\end{array}$ & $\begin{array}{l}\text { Karger } \\
\text { Open access }\end{array}$ \\
\hline $\begin{array}{l}\text { E-Mail k } \\
\text { www.ka }\end{array}$ & $\begin{array}{l}\text { This is an Open Access article licensed } \\
\text { Attribution-NonCommercial- } 4.0 \text { Inte } \\
\text { (http://www.karger.com/Services/Ope } \\
\text { the online version of the article only. } L \\
\text { mercial purposes requires written per }\end{array}$ & $\begin{array}{l}\text { the Creative Commons } \\
\text { al License (CC BY-NC) } \\
\text { sLicense), applicable to } \\
\text { d distribution for com- }\end{array}$ \\
\hline
\end{tabular}

Marcos M. Lima-Martínez

Endocrinology, Diabetes, Metabolism and Nutrition Unit

Anexo del Centro Médico Orinoco, Piso 2, Consultorio 30

Ciudad Bolívar (Venezuela)

E-Mail marcoslimamedical@ hotmail.com 


\section{Introduction}

The worldwide prevalence of diabetes was 425 million in 2017 and is predicted to rise to 629 million by 2045 [1]. In the South and Central America region, 24\% of adults with diabetes are undiagnosed, extending to $50 \%$ in some countries [2]. Diabetes was the fifth leading cause of death in Venezuela in 2012 [3]. Recent evidence has shown that the prevalence of diabetes and prediabetes in Venezuela is 8.3 and $14.6 \%$, respectively [4]. Considering the burden imposed by diabetes and the costs for its control, prevention represents a clear challenge for the health system.

Screening for type 2 diabetes mellitus (T2DM) and impaired glucose tolerance, with appropriate interventions, seems to be cost-effective [5]. The Finnish Diabetes Risk Score (FINDRISC) is one of the most efficient and widely used screening tools to detect new cases of T2DM [6]. However, FINDRISC needs to be validated in populations other than the original Finnish population for which it was developed, in order to determine performance attributes (e.g., sensitivity and specificity) [7].

FINDRISC includes anthropometric, metabolic, and lifestyle factors that predict T2DM [6]. By replacing the original cutoff point of waist circumference (WC) with the Latin American cutoff point in a Colombian (Bogotá) and Venezuelan (Barquisimeto) clinical setting, the modified FINDRISC (LA-FINDRISC) demonstrated a similar performance in identifying impaired glucose regulation in men and a better discriminative power in women than the original FINDRISC $[8,9]$; however, the optimal cutoff value for predicting glucose regulation disorders may be different depending on the population under study, even within the same country [7]. As there are no previous studies that have evaluated the usefulness of LA-FINDRISC as a screening tool for glucose regulation disorders in a population of the southeastern region of Venezuela, we decided to conduct the present study, the aim of which was to evaluate LA-FINDRISC as a screening tool for people with glucose metabolism disorder in Ciudad Bolívar, Venezuela.

\section{Subjects and Methods}

\section{Study Population}

This was an observational study using a test-versus-test modality. Ciudad Bolívar has approximately 203,190 inhabitants aged between 18 and 70 years. People in all the urban parishes of the city were invited to participate in screening sessions for cardiometabolic risk factors. We selected 200 subjects of both sexes who attended voluntarily and gave their informed consent to participate in the study. People with a previous diagnosis of diabetes mellitus, those hospitalized or with infectious diseases, and those using medications that could affect carbohydrate metabolism were not admitted. Figure 1 provides details on the sample selection and screening process.

\section{Clinical Evaluation}

Weight and height were measured with the subjects wearing only their underwear. The body mass index (BMI) was calculated as weight in kilograms divided by height in meters squared. WC was measured midway between the underside of the lowest rib and the iliac crest, in centimeters, with the subjects standing. Blood pressure, expressed in millimeters of mercury, was taken on the right arm, with the subjects in a sitting position after $10 \mathrm{~min}$ of rest, using the auscultation method with a conventional mercury sphygmomanometer.

\section{Biochemical Variables}

During 3 or more days before samples were taken, the patients maintained a free diet, without restriction on carbohydrate intake and performing the usual physical activities. With no less than $8 \mathrm{~h}$ of fasting, $5 \mathrm{~mL}$ of blood was drawn from the antecubital vein for a first determination of basal glucose. The blood was left to rest for $3 \mathrm{~min}$, after which it was centrifuged at 3,000 rpm; then, an aliquot of 500 Lambda was introduced into an automated Bayer Express Plus 500 device, using GLUCOSE PAP SL (ELITech Clinical Systems, Sées, France) as a reagent. Once the results were obtained, if patients met the required standard criterion (fasting blood glucose $<126 \mathrm{mg} / \mathrm{dL}$ ), they were given $75 \mathrm{~g}$ of anhydrous glucose (GLYCOLAB, Caracas, Venezuela) as indicated by the American Diabetes Association (ADA). The patients remained at rest for a period of 120 min without eating, drinking, or smoking. After $2 \mathrm{~h}$, a second blood sample was taken and the values were recorded.

\section{LA-FINDRISC Questionnaire}

The LA-FINDRISC questionnaire comprises eight variables: age, BMI, WC, physical activity, daily consumption of vegetables and fruits, antihypertensive drug use, personal history of hyperglycemia, and family history of diabetes. The WC cutoff values were adjusted for Latin America [8] by adding 4 points for subjects with abdominal obesity (WC $\geq 94 \mathrm{~cm}$ for men and $\geq 90 \mathrm{~cm}$ for women) and no points for those with normal WC values [9], the total score ranging from 0 to 26 points.

\section{Statistical Analysis}

Normally distributed continuous variables are presented as mean \pm standard deviation, and continuous variables with a nonnormal distribution are expressed as median and interquartile range. Categorical variables are presented as absolute number and percentage. Differences between categorical variables were determined through a $\chi^{2}$ test. A $t$ test was performed to assess differences between normally distributed continuous variables, and a Mann-Whitney U test for nonnormally distributed variables.

With the exception of height and BMI, all variables in this study revealed a nonnormal distribution. To obtain the LA-FINDRISC cutoff for predicting T2DM and impaired glucose regulation in this population, a ROC (receiver operating characteristic) curve was constructed. An area under the curve (AUC) of 1 was considered optimal, while an AUC $<0.5$ was considered signifying poor validity. The Youden index (YI) was used to determine the best 


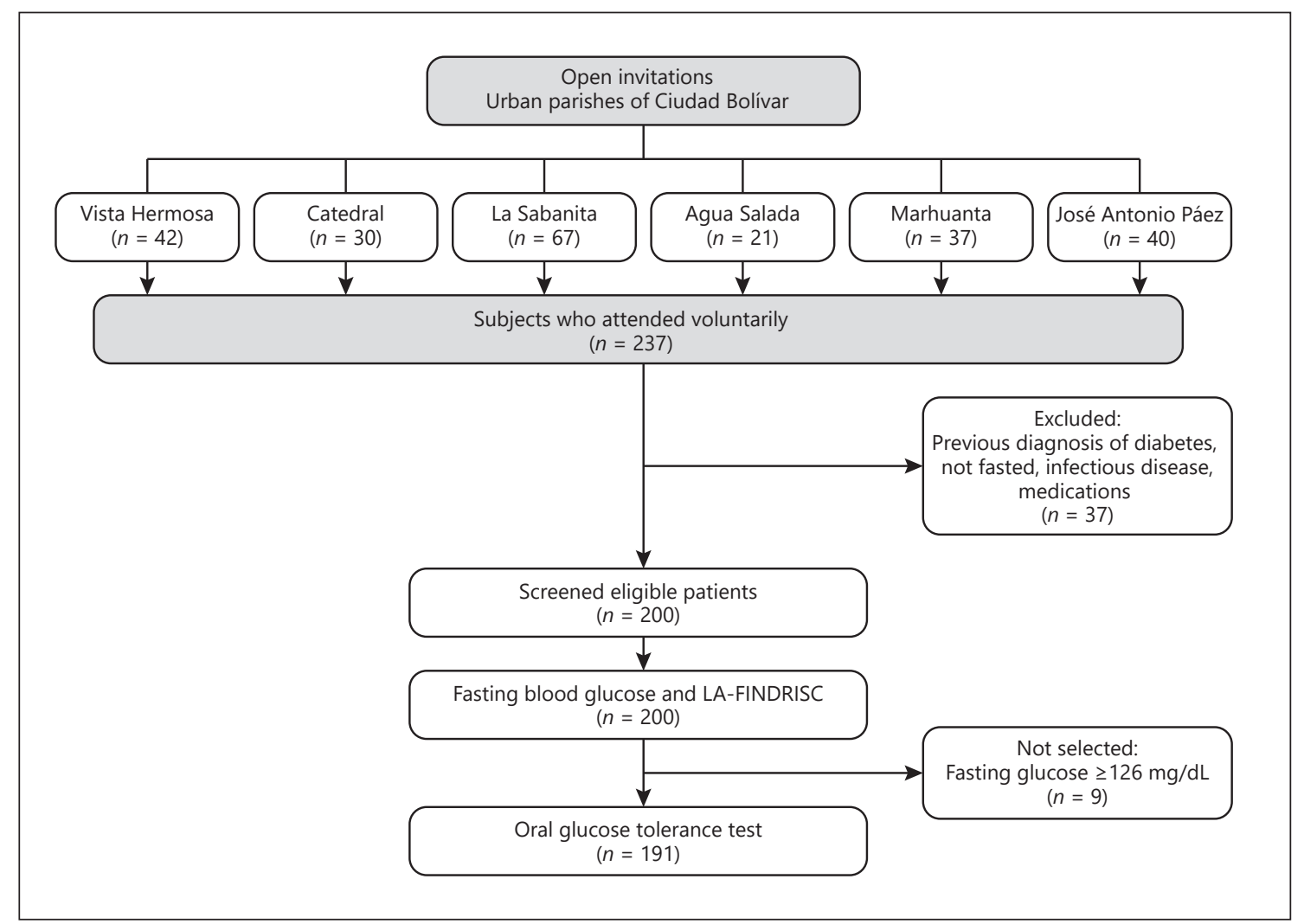

Fig. 1. Method of selection and screening tests.

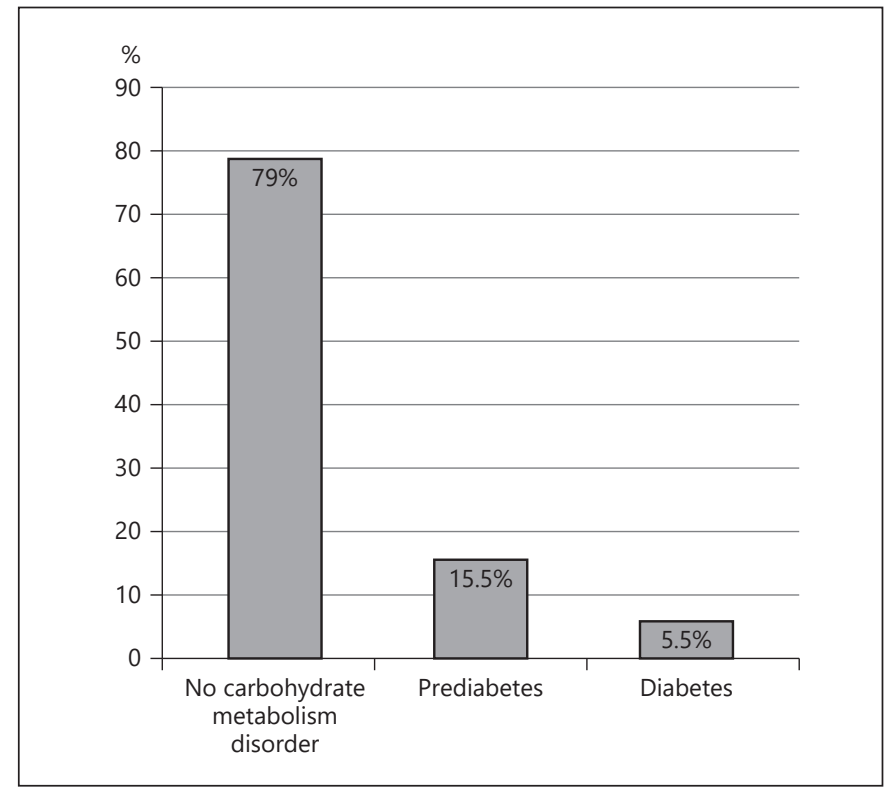

Fig. 2. Study population distribution according to the presence or absence of carbohydrate metabolism disorder. cutoff point from the ROC curve, calculated with the formula $\mathrm{YI}=$ (sensitivity + specificity) -1 . A $p$ value $<0.05$ was considered statistically significant. Statistical analysis was performed using SPSS version 20.0 software (SPSS Inc., Chicago, IL, USA).

\section{Results}

Table 1 shows the clinical characteristics of the subjects enrolled according to the presence or absence of carbohydrate metabolism disorder. A total of 200 subjects were evaluated, $64.5 \%$ female and $35.5 \%$ male, with a mean age of $35.20 \pm 13.84$ years. There were no significant differences in sex and height between the comparison groups. Age $(p=0.0001)$, weight $(p=0.024)$, BMI $(p=$ $0.005)$, and WC $(p=0.001)$ were significantly higher among those with carbohydrate metabolism disorder.

Figure 2 shows that $79 \%$ of the participants did not present with carbohydrate metabolism disorder, $15.5 \%$ had prediabetes, and 5.5\% had diabetes mellitus.

As expected, Table 2 shows significantly higher values of fasting blood glucose, LA-FINDRISC, and 2-h plasma glu- 
Table 1. Clinical characteristics according to the presence of carbohydrate metabolism disorder

\begin{tabular}{|c|c|c|c|c|}
\hline \multirow[t]{2}{*}{ Variables } & \multirow[t]{2}{*}{ Total $(n=200)$} & \multicolumn{2}{|c|}{ Carbohydrate metabolism disorder } & \multirow[t]{2}{*}{$p$ value } \\
\hline & & no $(n=158)$ & yes $(n=42)$ & \\
\hline \multicolumn{5}{|l|}{ Sex } \\
\hline Male & $71(35.5)$ & $54(34.2)$ & $17(40.5)$ & ns \\
\hline Female & $129(64.5)$ & $104(65.8)$ & $25(59.5)$ & \\
\hline Age, years & $34.50(22.00-45.75)$ & $30.00(21.00-42.00)$ & $44.00(33.75-59.25)$ & 0.0001 \\
\hline Age range, years & $18-68$ & $18-68$ & $18-62$ & \\
\hline Weight, kg & $65.65(56.07-79.27)$ & $64.90(54.87-76.82)$ & $71.40(61.30-84.27)$ & 0.024 \\
\hline Height, m & $1.62 \pm 0.08$ & $1.62 \pm 0.08$ & $1.62 \pm 0.09$ & ns \\
\hline $\mathrm{BMI}, \mathrm{kg} / \mathrm{m}^{2}$ & $25.92 \pm 5.71$ & $25.40 \pm 5.62$ & $27.88 \pm 5.69$ & 0.005 \\
\hline $\mathrm{WC}, \mathrm{cm}$ & $88.00(77.00-96.00)$ & $86.00(76.00-94.00)$ & $92.95(85.00-101.25)$ & 0.001 \\
\hline
\end{tabular}

Values are presented as $n(\%)$, mean $\pm \mathrm{SD}$, or median (IQR). BMI, body mass index; WC, waist circumference.

Table 2. Fasting glucose, post-load glucose, and LA-FINDRISC according to the absence of presence of carbohydrate metabolism disorder

\begin{tabular}{|c|c|c|c|}
\hline & \multicolumn{2}{|c|}{ Carbohydrate metabolism disorder } & \multirow[t]{2}{*}{$p$ value } \\
\hline & no & yes & \\
\hline & $(n=158)$ & $(n=42)$ & \\
\hline Fasting glucose, $\mathrm{mg} / \mathrm{dL}$ & $82.00(76.00-89.00)$ & $102.00(93.50-120.5)$ & 0.0001 \\
\hline LA-FINDRISC & $8.00(5.00-12.00)$ & $13.50(8.00-18.00)$ & 0.0001 \\
\hline 2-h plasma glucose, $\mathrm{mg} / \mathrm{dL}$ & $\begin{array}{l}(n=167) \\
90.00(80.75-98.00)\end{array}$ & $\begin{array}{l}(n=24) \\
156.00(120.75-185.25)\end{array}$ & 0.0001 \\
\hline
\end{tabular}

cose in the group of patients with carbohydrate metabolism disorder when compared to those without it $(p=0.0001)$.

Table 3 shows the components of LA-FINDRISC according to the presence or absence of carbohydrate metabolism disorder. Age $(p=0.0001), \mathrm{BMI}(p=0.011)$, and WC $(p=0.031)$ were significantly higher among the subjects with carbohydrate metabolism disorder when compared to those without it. Similarly, we found a significantly higher number of sedentary $(p=0.039)$ and hypertensive subjects $(p=0.0001)$, as well as those with a history of glucose $>100 \mathrm{mg} / \mathrm{dL}$ ( $p=0.0001$ ), in the carbohydrate metabolism disorder group. There were no significant differences in family history of diabetes and fruit consumption between the comparison groups.

Figure 3 shows a good AUC of 0.727 (95\% CI: $0.636-$ $0.818, p=0.0001$ ). A LA-FINDRISC cutoff value of 14 predicted a high risk of diabetes mellitus or carbohydrate metabolism disorder, with a sensitivity of $45.2 \%$, a specificity of $89.9 \%$, a positive predictive value of $54.3 \%$, and a negative predictive value of $86.1 \%$ (YI: 0.351 ). A LA-FINDRISC cutoff value of 10 showed a sensitivity of $66.7 \%$, a specificity of $66.5 \%$, a positive predictive value of $34.6 \%$, and a negative predictive value of $88.2 \%$ (YI: 0.331 ).

Using the best cutoff value obtained from LA-FINDRISC (Fig. 4), we observed a significantly higher percentage of subjects with LA-FINDRISC $>14$ points among those with carbohydrate metabolism disorder than among those without it ( 45.2 vs. $10.1 \%, p=0.0001$ ). An individual presenting with a LA-FINDRISC $>14$ points has a 7.3 times higher risk of carbohydrate metabolism disorder than a person with a lower score (95\% CI: 3.30-16.27).

\section{Discussion}

The most significant findings of our study were the following: (1) a LA-FINDRISC cutoff value $>14$ points predicts carbohydrate metabolism disorder with a sensitivity 


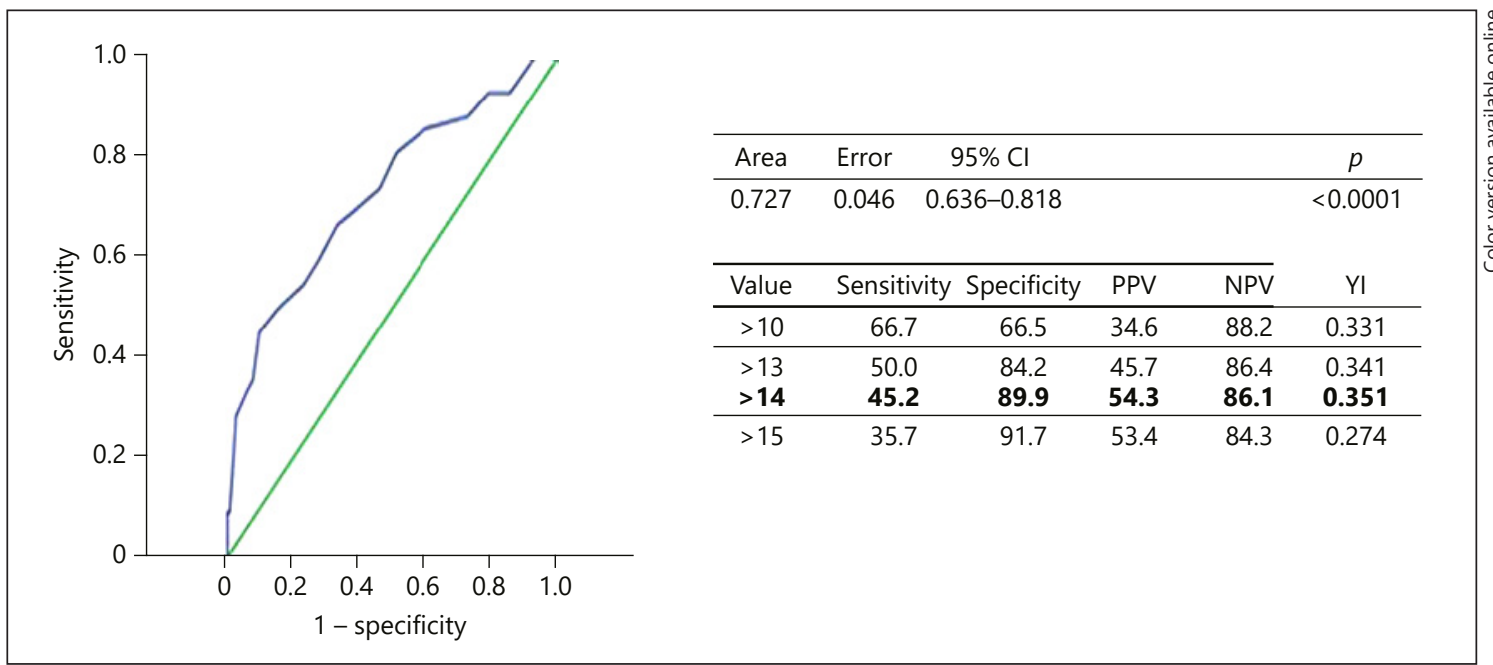

Fig. 3. Receiver operating characteristic curve for LA-FINDRISC to predict impaired glucose regulation in the study population. PPV, positive predictive value; NPV, negative predictive value; YI, Youden index.

Table 3. LA-FINDRISC components according to the absence or presence of carbohydrate metabolism disorder

\begin{tabular}{|c|c|c|c|c|c|}
\hline Variables & $\begin{array}{l}\text { Score, } \\
\text { points }\end{array}$ & $\begin{array}{l}\text { Total } \\
(n=200)\end{array}$ & $\begin{array}{l}\text { No } \\
(n=158)\end{array}$ & $\begin{array}{l}\text { Yes } \\
(n=42)\end{array}$ & $p$ value \\
\hline \multicolumn{6}{|l|}{ Age } \\
\hline$<35$ years & 0 & $98(49.0)$ & $86(54.4)$ & $12(28.6)$ & \multirow{5}{*}{0.0001} \\
\hline $35-44$ years & 1 & $52(26.0)$ & $41(25.9)$ & $11(26.2)$ & \\
\hline $45-54$ years & 2 & $28(14.0)$ & $23(14.6)$ & $5(11.9)$ & \\
\hline $55-64$ years & 3 & $17(8.5)$ & $7(4.4)$ & $10(23.8)$ & \\
\hline$>64$ years & 4 & $5(2.5)$ & $1(0.6)$ & $4(9.5)$ & \\
\hline \multicolumn{6}{|l|}{ Family history of diabetes } \\
\hline No & 0 & $73(36.5)$ & $56(35.4)$ & $17(40.5)$ & \multirow[t]{3}{*}{ ns } \\
\hline Grandparents, uncles, cousins & 3 & $74(37.0)$ & $61(38.6)$ & $13(31.0)$ & \\
\hline Parents, children, siblings & 5 & $53(26.5)$ & $41(25.9)$ & $12(28.6)$ & \\
\hline \multicolumn{6}{|l|}{ Physical activity } \\
\hline Yes & 0 & $40(20.0)$ & $36(22.8)$ & $4(9.5)$ & \multirow[t]{2}{*}{0.039} \\
\hline No & 2 & $160(80.0)$ & $122(77.2)$ & $38(90.5)$ & \\
\hline \multicolumn{6}{|l|}{ Daily fruit intake } \\
\hline Yes & 0 & $38(19.0)$ & $28(17.7)$ & $10(23.8)$ & \multirow[t]{2}{*}{ ns } \\
\hline No & 1 & $162(81.0)$ & $130(82.3)$ & $32(76.2)$ & \\
\hline \multicolumn{6}{|l|}{ WC } \\
\hline $\mathrm{F}:<90 \mathrm{~cm} ; \mathrm{M}:<94 \mathrm{~cm}$ & 0 & $115(57.5)$ & $97(61.4)$ & $18(42.9)$ & \multirow[t]{2}{*}{0.031} \\
\hline $\mathrm{F}: \geq 90 \mathrm{~cm} ; \mathrm{M}: \geq 94 \mathrm{~cm}$ & 4 & $85(42.5)$ & $61(38.6)$ & $24(57.1)$ & \\
\hline \multicolumn{6}{|l|}{ BMI } \\
\hline$<25 \mathrm{~kg} / \mathrm{m}^{2}$ & 0 & $98(49.0)$ & $86(54.4)$ & $12(28.6)$ & \multirow[t]{3}{*}{0.011} \\
\hline $25-30 \mathrm{~kg} / \mathrm{m}^{2}$ & 1 & $56(28.0)$ & $40(25.3)$ & $16(38.1)$ & \\
\hline$>30 \mathrm{~kg} / \mathrm{m}^{2}$ & 2 & $46(23.0)$ & $32(20.3)$ & $14(33.3)$ & \\
\hline \multicolumn{6}{|l|}{ HBP } \\
\hline No & 0 & $161(80.5)$ & $137(86.7)$ & $24(57.1)$ & \multirow[t]{2}{*}{0.0001} \\
\hline Yes & 2 & $39(19.5)$ & $21(13.3)$ & $18(42.9)$ & \\
\hline \multicolumn{6}{|l|}{ Glucose $>100 \mathrm{mg} / \mathrm{dL}$} \\
\hline No & 0 & $164(82.0)$ & $141(89.2)$ & $23(54.8)$ & \multirow[t]{2}{*}{0.0001} \\
\hline Yes & 5 & $36(18.0)$ & $17(10.8)$ & $19(45.2)$ & \\
\hline
\end{tabular}

BMI, body mass index; HBP, high blood pressure; WC, waist circumference. 
of $45.2 \%$ and a specificity of $89.9 \%$ and with an AUC of 0.72 , and (2) this cutoff value identifies individuals with a 7.3 times higher risk of carbohydrate metabolism disorder.

The oral glucose tolerance test (OGTT) is considered the gold standard for the diagnosis of T2DM, but it has disadvantages as a population screening tool because it is expensive, takes a long time to perform, and is uncomfortable for patients. In contrast, FINDRISC assessment does not require blood samples; subjects just need to answer eight questions. However, this questionnaire was designed for the population of Finland. Thus, it needs to be validated in other populations [7].

The FINDRISC questionnaire was constructed with data from a Finnish population cohort of subjects between 35 and 64 years of age randomly selected in 1987 and followed up for 10 years, in order to predict the development of diabetes. It was validated with another independent cohort randomly selected in 1992 and followed up for 5 years [6]. The FINDRISC varied between 0 and 20 points, and a value $\geq 9$ points predicted diabetes with a sensitivity of 78 and $81 \%$ and a specificity of 77 and 76\% in the 1987 and 1992 cohorts, respectively [6].

In Spain, FINDRISC validation was carried out based on population data representative of a locality in southern Spain for which both the prevalence and the incidence of T2DM were known [10]. It was found that FINDRISC showed good results in detecting undiagnosed cases of T2DM (AUC $=0.74)$ and in predicting new-onset diabetes $(A U C=0.75)$. The best risk prediction of new-onset T2DM was found for subjects with a FINDRISC $\geq 9$ [11]. Similarly, a study in Greece validated a FINDRISC $\geq 15$, which had a sensitivity of $82 \%$ and a specificity of $60 \%$ to predict unknown diabetes. Similar to our study, the AUC obtained was 0.72 for detecting diabetes or glucose metabolism disorder [12].

In Italy, Franciosi et al. [13] reported that a FINDRISC $\geq 9$ had a sensitivity of $77 \%$, a specificity of $45 \%$, and an AUC of 0.67 (95\% CI: $0.64-0.70$ ) for the detection of T2DM [13]. Unlike our study, that by Franciosi et al. [13] enrolled individuals between 55 and 75 years of age, based on their cardiovascular risk profile, which can be considered a selection bias.

In Germany, a cross-sectional study (KORA Survey 2000) [14] evaluated the association between the risk scores of 4 questionnaires, including FINDRISC, and the presence of undiagnosed T2DM in 1,353 participants aged 55-74 years. The questionnaires showed a low positive predictive value and a high negative predictive value, so they are useful instruments for ruling out risk, but not so much for confirming it - similar to what we found

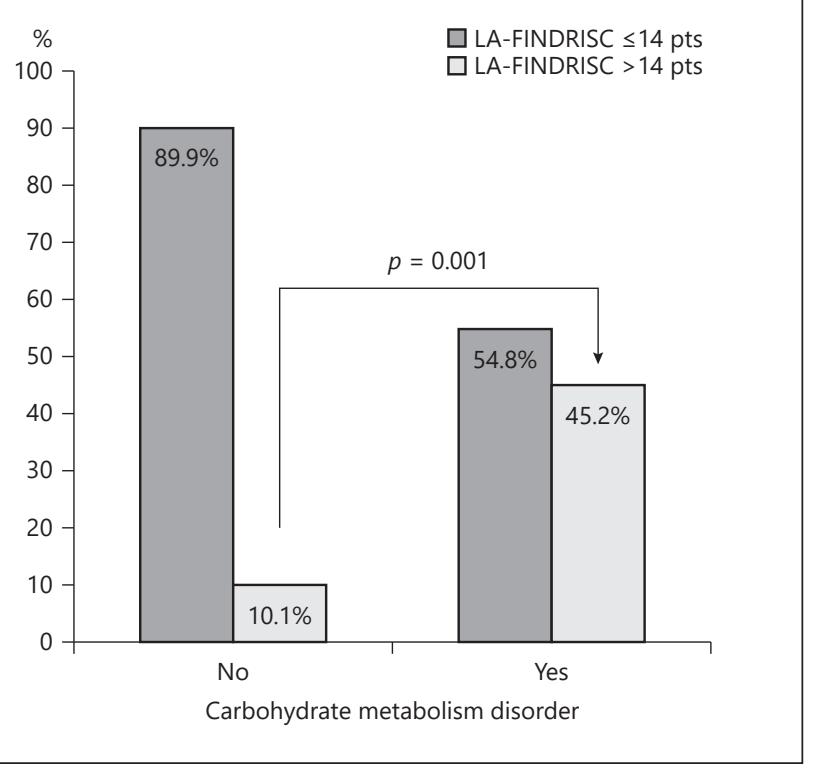

Fig. 4. Carbohydrate metabolism disorder according to LA-FINDRISC cutoff value ( $p=0.0001$; odds ratio: 7.332 ; 95\% CI: $3.302-$ 16.279).

in our study, where the positive predictive value was $54.3 \%$ and the negative predictive value $86 \%$. The authors concluded that their results could be due to the fact that the evaluated questionnaires have low validity when applied to populations for which they had not been designed.

In order to improve FINDRISC validity in the Latin American population, the original questionnaire was modified, incorporating WC cutoff points validated in the Latin American population to diagnose abdominal obesity [8]. The first LA-FINDRISC validation study was performed in Colombia, on 421 subjects residing in Bogotá, by the Colombian Diabetes Association [9]. A sensitivity of $74 \%$ and a specificity of $60 \%$ were obtained for detecting any glucose metabolism disorder (diabetes or prediabetes) with a score $>12$ points [9].

On the other hand, LA-FINDRISC was also evaluated in two primary care centers in Uruguay, where a total of 109 subjects were included and it was shown that a score $>14$ had a sensitivity of $70 \%$ and a specificity of $66 \%$ for predicting unknown diabetes or prediabetes [7]. Although the cutoff point found in this study is similar to ours, the differences in sensitivity may be due to the fact that the subjects evaluated in Uruguay had been enrolled from clinical centers and therefore are not necessarily representative of the general population. In addition, the popula- 


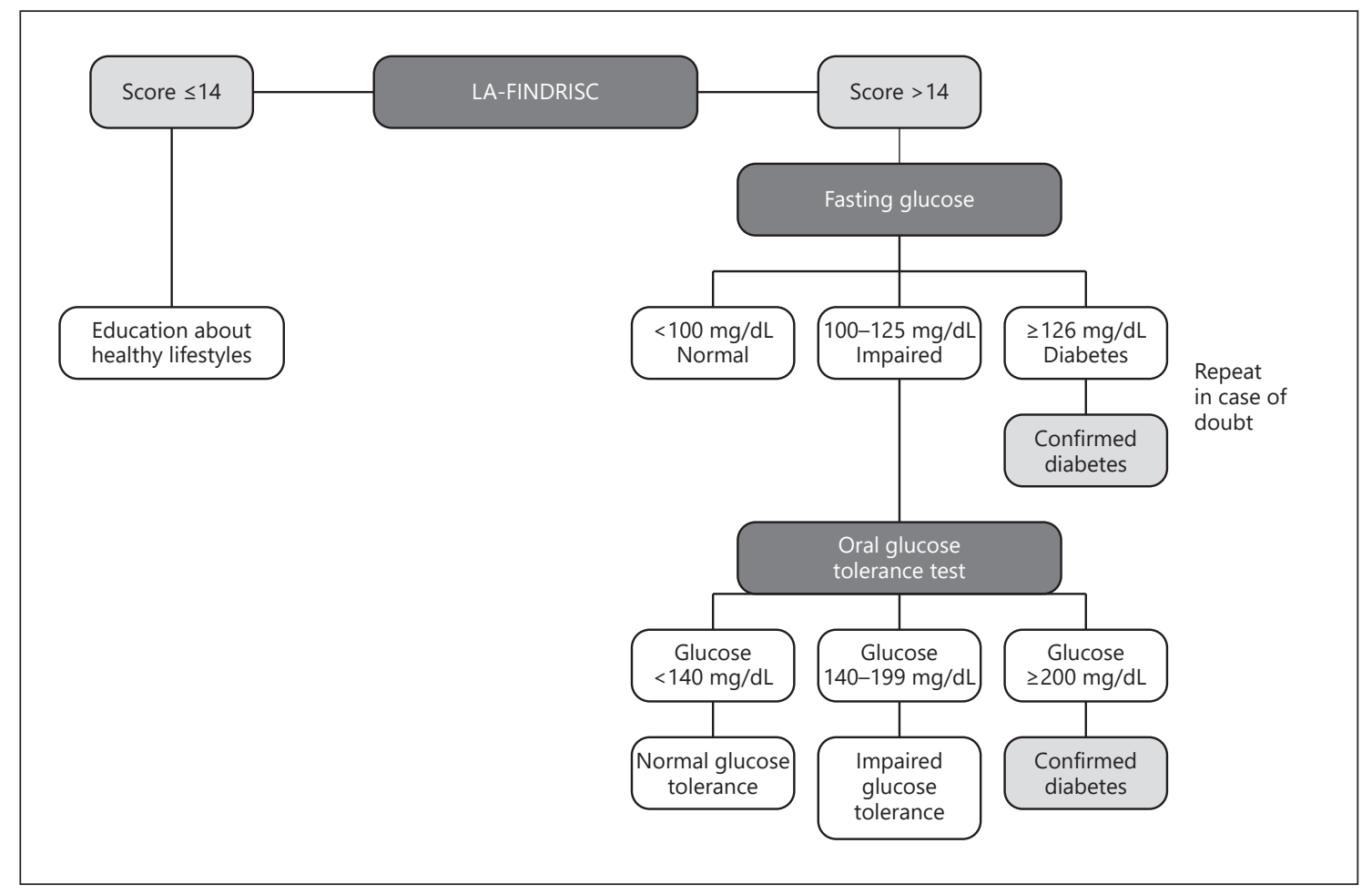

Fig. 5. Proposed algorithm for the screening and diagnosis of type 2 diabetes mellitus in Ciudad Bolívar, Venezuela.

tion evaluated in our study was younger (mean age: 34.50 years), thinner (mean BMI: $25.92 \pm 5.71 \mathrm{~kg} / \mathrm{m}^{2}$ ), and therefore healthier, a fact that might have had a bearing on the low sensitivity found with the same cutoff point. There has been demonstrated an association between overweight/obesity and the prevalence of impaired glucose regulation [15].

Similarly, a first validation study conducted in Venezuela [9], which included 334 subjects selected from an obesity and cardiometabolic risk factor clinic located in Barquisimeto, evidenced that a LA-FINDRISC $>14$ had a sensitivity of 97 and $91 \%$ and a specificity of 70 and $78 \%$ for predicting glucose metabolism impairment in men and women, respectively. On the other hand, a random sample of 521 participants recruited from the EVESCAM study showed that a LA-FINDRISC $>10$ had a sensitivity of $63 \%$ and a specificity of $61 \%$ for predicting glucose metabolism impairment, with an AUC of 0.68 [7].

As all these studies show, FINDRISC and LA-FINDRISC predictive accuracy varies from one population to another, and the best cutoff value for screening may depend on the characteristics of the population. For ex- ample, the cutoff value could be higher for subjects with a greater number of risk factors. We also emphasize that selection of the best cutoff value may depend on the purpose of the screening: if it is done to select candidates for a community diabetes prevention program, then a lower cutoff point may be preferable in order to favor sensitivity over specificity. In fact, in our study, it was observed that a LA-FINDRISC $>10$ points had a sensitivity of $66.7 \%$ and a specificity of $66.5 \%$ for predicting carbohydrate metabolism disorder, similar to the results observed in the EVESCAM study [7]. In contrast, if the prevention program is limited and one wishes to select only very-high-risk subjects, it is preferable to have a higher cutoff value ( $>14$ points), which has a higher specificity.

Although the current study provides interesting findings, it is necessary to recognize some limitations. Firstly, the sample size is relatively small; a larger sample would also allow for multivariate statistical methods to be employed, instead of bivariate statistical testing only. However, the statistical power was sufficient to detect significant differences in the studied variables. In addition, FINDRISC and LA-FINDRISC validation studies that have a 
smaller sample size have been reported in the literature [16]. Secondly, we did not have a randomly or stratified selected sample; however, all subjects were recruited by open invitation with screening purposes from all the urban parishes of Ciudad Bolívar, similar to the LA-FINDRISC validation study performed in Bogotá by the Colombian Diabetes Association [9]. Thirdly, the study has a crosssectional and not a longitudinal design, which does not allow us to affirm that subjects with a score $>14$ will have a higher incidence of T2DM; however, we emphasize that previous studies conducted on the same population have shown a strong association between LA-FINDRISC $>14$ points and other metabolic risk factors [17, 18].

We suggest the application of the LA-FINDRISC questionnaire as part of a diabetes detection and prevention program for subjects older than 18 years in all primary care centers of Ciudad Bolívar, Venezuela (Fig. 5). Those subjects having a LA-FINDRISC $>14$ points should be tested for fasting plasma glucose as the first diagnostic test. If glucose levels are between 100 and $125 \mathrm{mg} / \mathrm{dL}$ (impaired fasting glucose), an OGTT is in order as a second diagnostic test. A fasting glucose $\geq 126 \mathrm{mg} / \mathrm{dL}$ requires that the test be repeated, as suggested by the ADA. An OGTT yielding a 2 -h glucose value $<140 \mathrm{mg} / \mathrm{dL}$ is considered normal. In contrast, a reading between 140 and $199 \mathrm{mg} / \mathrm{dL}$ is indicative of impaired glucose tolerance (prediabetes), and glucose $\geq 200 \mathrm{mg} / \mathrm{dL}$ confirms diabetes. In case of prediabetes, a change in lifestyle (diet plus exercise) should always be encouraged as a beneficial course of action [19].

\section{Conclusions}

Based on our results, we conclude that a LA-FINDRISC $>14$ points has low sensitivity but high specificity for predicting carbohydrate metabolism disorders (prediabetes or diabetes) in this group of patients from Ciudad Bolívar, Venezuela. These results suggest that the number of false negatives could be high and the number of false positives associated with this test decreases.

\section{Acknowledgements}

We would like to thank Prof. Carlos Mota for critically reviewing the manuscript.

\section{Statement of Ethics}

The subjects gave their written informed consent. The study protocol was approved by the research institute's Committee on Human Research.

\section{Disclosure Statement}

The authors have no conflicts of interest to declare.

\section{References}

1 International Diabetes Federation. IDF Diabetes Atlas, 8th edition [Internet] [accessed 2018 Apr 22]. IDF; 2017. http://www.diabetesatlas.org/.

2 Aschner P, Aguilar-Salinas C, Aguirre L, Franco L, Gagliardino JJ, de Lapertosa SG, et al.; IDF Diabetes Atlas. Diabetes in South and Central America: an update. Diabetes Res Clin Pract. 2014 Feb;103(2):238-43.

3 Nieto-Martínez R, González-Rivas JP, LimaMartínez M, Stepenka V, Rísquez A, Mechanick JI. Diabetes Care in Venezuela. Ann Glob Health. 2015 Nov-Dec;81(6):776-91.

4 Nieto-Martínez R, Mechanick JI, Brajkovich I, Ugel E, Risques A, Florez H, et al. Prevalence of diabetes in three regions of Venezuela. The VEMSOLS study results. Prim Care Diabetes. 2018 Apr;12(2):126-32.

Validation of FINDRISC for Latin America
5 Gillies CL, Lambert PC, Abrams KR, Sutton AJ, Cooper NJ, Hsu RT, et al. Different strategies for screening and prevention of type 2 diabetes in adults: cost effectiveness analysis. BMJ. 2008 May;336(7654):1180-5.

6 Lindström J, Tuomilehto J. The diabetes risk score: a practical tool to predict type 2 diabetes risk. Diabetes Care. 2003 Mar;26(3):725-31.

7 Nieto-Martínez R, González-Rivas JP, Aschner P, Barengo NC, Mechanick JI. Transculturalizing diabetes prevention in Latin America. Ann Glob Health. 2017 May-Aug;83(3-4): 432-43.

8 Aschner P, Buendía R, Brajkovich I, Gonzalez A, Figueredo R, Juarez XE, et al. Determination of the cutoff point for waist circumference that establishes the presence of abdominal obesity in Latin American men and women. Diabetes Res Clin Pract. 2011 Aug;93(2):243-7.
9 Aschner P, Nieto-Martínez R, Marin A, Ríos $\mathrm{M}$. Validation of the FINDRISC score as a screening tool for people with impaired glucose regulation in Latin America using modified regional score points for waist circumference according to the validated regional cutoff values for abdominal obesity. Minerva Endocrinol. 2012;37:114.

10 Soriguer F, Rojo-Martínez G, Almaraz MC, Esteva I, Ruiz de Adana MS, Morcillo S, et al. Incidence of type 2 diabetes in southern Spain (Pizarra Study). Eur J Clin Invest. 2008 Feb; 38(2):126-33.

11 Soriguer F, Valdés S, Tapia MJ, Esteva I, Ruiz de Adana MS, Almaraz MC, et al. [Validation of the FINDRISC (FINnish Diabetes RIsk SCore) for prediction of the risk of type 2 diabetes in a population of southern Spain. Pizarra Study]. Med Clin (Barc). 2012 Apr;138(9):371-6. 
12 Makrilakis K, Liatis S, Grammatikou S, Perrea $\mathrm{D}$, Stathi C, Tsiligros $\mathrm{P}$, et al. Validation of the Finnish Diabetes Risk Score (FINDRISC) questionnaire for screening for undiagnosed type 2 diabetes, dysglycaemia and the metabolic syndrome in Greece. Diabetes Metab. 2011 Apr;37(2):144-51.

13 Franciosi M, De Berardis G, Rossi MC, Sacco $\mathrm{M}$, Belfiglio M, Pellegrini F, et al. Use of the diabetes risk score for opportunistic screening of undiagnosed diabetes and impaired glucose tolerance: the IGLOO (Impaired Glucose Tolerance and Long-Term Outcomes Observational) study. Diabetes Care. 2005 May;28(5): 1187-94.
14 Rathmann W, Martin S, Haastert B, Icks A, Holle R, Löwel H, et al.; KORA Study Group. Performance of screening questionnaires and risk scores for undiagnosed diabetes: the KORA Survey 2000. Arch Intern Med. 2005 Feb;165(4):436-41.

15 Alattar A, Al-Majed H, Almuaili T, Almutairi O, Shaghouli A, Altorah W. Prevalence of impaired glucose regulation in asymptomatic Kuwaiti young adults. Med Princ Pract. 2012;21(1):51-5.

16 Pereira de Moura B, Do Santos Amorim PR, Castro Franceschini SC. Validation of a screening tool for identifying Brazilians with impaired glucose tolerance. Int J Diabetes Dev Ctries. 2012;32(3):116-21.
17 Lima-Martínez MM, Arrau C, Jerez S, Paoli M, González-Rivas JP, Nieto-Martínez R, et al. Relationship between the Finnish Diabetes Risk Score (FINDRISC), vitamin D levels, and insulin resistance in obese subjects. Prim Care Diabetes. 2017 Feb;11(1):94-100.

18 Lima-Martínez MM, Colmenares L, Campanelli Y, Paoli M, Rodney M, Santos RD, et al. Epicardial adipose tissue thickness and type 2 diabetes risk according to the FINDRISC modified for Latin America. Clin Investig Arterioscler. 2019 Jan-Feb;31(1):15-22.

19 Alefishat EA, Abu Farha RK, Al-Debei MM Self-reported adherence among individuals at high risk of metabolic syndrome: effect of knowledge and attitude. Med Princ Pract. 2017;26(2):157-63. 